\title{
The relationship between coping strategies and sleep problems: The role of depressive symptoms
}

Malgorzata W. Kozusznik ${ }^{12}$, Sara Puig-Perez ${ }^{3}$, Barbara Kożusznik ${ }^{4}$, Matias M. Pulopulos ${ }^{5}$

${ }^{1}$ Faculty of Psychology, Complutense University of Madrid, Madrid, Spain

${ }^{2}$ Research Group Organizational and Occupational Psychology and Professional Learning, KU

Leuven, Belgium

${ }^{3}$ Research Group for Psychology and Quality of Life (PsiCal), Valencian International University, Valencia, Spain

${ }^{4}$ Institute of Psychology, University of Silesia in Katowice, Katowice, Poland

${ }^{5}$ Department of Experimental Clinical and Health Psychology, Ghent University, Ghent, Belgium

Corresponding author: Matias M. Pulopulos - matias.pulopulos@ugent.be 


\begin{abstract}
Background: Several studies have proposed that coping strategies are a key predictor of sleep problems. Furthermore, some authors have suggested that depressive symptoms, a factor that is related to both coping strategies and sleep, may play a critical role in this relationship. However, this preliminary research has shown mixed results.

Purpose: The aim of this research is to study the relationship between coping strategies (i.e., emotion-focused and problem-focused coping) and sleep, and investigate whether this relationship is direct or mediated by depressive symptoms.
\end{abstract}

Methods: Using a cross-sectional design, we tested this idea in a sample of 723 participants from the Midlife in the United States 2 study (mean age=54.22 years, age range $=25-74$ years, $54.40 \%$ females, $95.1 \%$ had at least a high school education). We applied mediation analyses with bootstrapped bias-corrected $95 \%$ confidence intervals to test total, direct, and indirect effects. Coping and depression were assessed using questionnaires. Objective and subjective sleep quantity and quality were measured using the Pittsburgh Sleep Quality Index (PSQI), sleep diaries, and actigraphy.

Results: The results show that low emotion-focused coping and high problem-focused coping are associated with lower depressive symptoms, which, in turn, are associated with better objective and subjective sleep quality. Moreover, greater use of emotion-focused coping is related to more perceived sleep time.

Conclusions: This study sheds light on the process of the development of sleep problems in people who use different coping strategies. It offers explanations for the association between emotion-focused and problem-focused coping and sleep problems, via depressive symptoms. Keywords: Emotion-focused coping, Problem-focused coping, Sleep, Depression 


\section{Introduction}

Sleep is a complex, multidimensional behavior that has an impact on both psychological and physical health $[1,2]$. Inadequate levels of sleep dimensions, particularly sleep duration and quality, are associated with important health problems such as cognitive deficits [3], cardiovascular diseases [4], and endocrine dysregulations such as obesity [5]. Although sleep has been found to be influenced by different psychological processes $[6,7]$, the role these factors play in sleep quality and quantity has not yet been fully explained. Understanding the way different factors contribute to differences in sleep is crucial to preventing and treating sleep problems and sleep-related health disorders.

Previous research suggests that coping styles may be important in understanding interindividual differences in sleep [8]. Coping can be defined as "volitional efforts to regulate emotions, thoughts, behaviors, physiology, and the environment in stressful events or circumstances" [9], and it is relatively stable over time [10]. The literature identifies two major groups of coping strategies: problem-focused coping refers to one's efforts to improve the stressful situation, solve the problem [11], or decrease the threat [12], whereas emotionfocused coping aims to lessen the emotional discomfort triggered by the situation $[13,14]$ and regulate the distressing emotional and physiological reactions to the stressor [11]. Although the effectiveness of certain coping strategies can be context dependent [15], palliative or emotionfocused coping strategies are generally considered dysfunctional or "maladaptive" [16]. Specifically, even though emotion-focused coping may be useful when responding to uncontrollable stressors or major life events $[17,18]$, if some degree of control over the situation is possible, problem-focused coping turns out to be effective [19]. Furthermore, in contrast to problem-focused coping, emotion-focused coping does not reduce discrepancies; therefore, this type of coping is generally not considered helpful in dealing with stress 
$[13,14,20,21]$. Regarding the relationship between coping styles and sleep, in the Psycho-biobehavioral model of vulnerability to insomnia, Harvey, Gehrman and Espie [8] recently proposed that emotion-focused coping may be a risk factor for developing sleep problems. Along these lines, empirical evidence supports the idea that emotion-focused coping strategies are associated with sleep disruption [8,22-25]. For instance, Sadeh, Keinan and Dahon [23] showed that a high emotion-focused coping score was a predictor of reduced sleep time (as measured by sleep diaries and actigraphy) in a group of good sleeping students during the week before an important interview. Moreover, Fernandez-Mendoza and colleagues [25] revealed a positive association between emotion-focused coping and vulnerability in developing insomnia, suggesting that maladaptive coping strategies (such as emotion-focused coping) predispose people to the development of insomnia.

Although these studies seem to support the idea that emotion-focused coping is related to sleep problems, it is important to note that maladaptive coping has been strongly associated with other psychological conditions that also affect sleep. Within this context, depression may be a critical factor in understanding the relationship between coping and sleep problems. On the one hand, theoretical and empirical research indicates that maladaptive coping strategies are important factors leading to the development of depression [9, 26-33]. Furthermore, longitudinal studies have demonstrated that the use of emotion-focused coping strategies favors the development of depression in different populations [e.g., 34-37]. On the other hand, research shows that depression contributes to higher sleep disturbance, measured with the Pittsburgh Sleep Quality Index (PSQI) [38]. In addition, insomnia disorder frequently co-occurs with mental health disorders such as Major Depressive Disorder [39], and studies have shown that up to $80 \%$ of individuals with Major Depressive Disorder also present symptoms of insomnia [40]. Therefore, an important question is whether emotion-focused coping strategies 
may lead to sleep problems due to their relationship with depression (i.e., a mediating path from coping to sleep through depression). Along these lines, in a two-wave study with 55 cancer patients, Hoyt and colleagues [24] showed that depressive symptoms mediated the relationship between avoidance coping and sleep symptom interference (e.g., the degree of interference with daily functioning, the appearance of sleep problems to others, and worries related to sleep problems). They observed that more avoidance coping was related to more depressive symptoms, leading to more sleep symptom interference. Thomas and colleagues [41] also made an attempt to test the mediating role of depressive symptoms between coping (i.e., approach, avoidance) and perceived sleep, but they failed to observe a significant relationship in a sample of 23 prostate cancer and 33 breast cancer patients. Therefore, so far, research investigating the mediating role of depression in the relationship between coping and sleep is scarce and has shown mixed results. However, these studies $[23,40]$ had small samples and only measured subjective sleep parameters. Thus, a critical gap in the literature is to determine whether emotion-focused coping is directly related to sleep, or whether depression mediates the relationship between emotion-focused coping and sleep.

In addition to the maladaptive emotion-focused coping strategy studied by Harvey and colleagues [8], sleep might also be influenced by the use of more adaptive coping strategies such as problem-focused coping. So far, this relationship has hardly been investigated. Sadeh and colleagues [23] found a positive association between problem-focused coping and sleep quantity measures (i.e., sleep period and true sleep time). Moreover, Thomas and colleagues [41] showed a positive relationship between approach coping and better sleep in prostate cancer patients. These studies support the link between problem-focused coping and sleep. However, they included small and homogeneous samples (36 healthy students in Sadeh and colleagues [23] and 56 cancer patients in Thomas and colleagues [41]. Therefore, more 
research with a larger community sample is needed to investigate the role of problem-focused coping in sleep in greater depth. Moreover, apart from its relationship with sleep, longitudinal studies have shown that problem-focused coping predicts changes in depression [42-44], suggesting that depression may also play a role in the relationship between problem-focused coping and sleep.

Drawing on the above, and based on the Psycho-bio-behavioral model of vulnerability to insomnia [8], coping strategies seem to be key factors in understanding interindividual variability in sleep. Moreover, depressive symptoms, which are strongly related to both coping strategies and sleep, may be a crucial factor in these relationships. However, no research has addressed the possibility of a mediation path from coping through depression to sleep in a large sample, considering key dimensions of coping (i.e., emotion- and problem-focused) and different facets of sleep (i.e., sleep duration, sleep quality), and using different measures of sleep (i.e., general sleep, perceived sleep using a diary, and actigraphy). Therefore, the aim of this cross-sectional study was to investigate the relationship between coping strategies (emotion-focused coping and problem-focused coping) and sleep problems (quantity and quality) in a large sample, using objective and subjective measures of sleep quantity and quality (general sleep using a questionnaire, perceived sleep using a diary, and actigraphy). Specifically, we aimed to study whether the relationship between coping and sleep problems is direct or mediated by depressive symptoms. Following the Psycho-bio-behavioral model of vulnerability to insomnia [8], we expected emotion-focused coping to be related to more sleep problems. Moreover, given that depression is related to both coping strategies and sleep, we hypothesized that part of the variance in sleep problems explained by coping strategies could be due to differences in depressive symptoms (i.e., a mediating path from coping to sleep 
through depression). Finally, we investigate whether problem-focused coping is related to sleep, and whether this relationship is also mediated by depressive symptoms.

\section{Method}

\section{Participants}

The study sample was recruited as part of the Midlife in the United States 2 study (MIDUS 2), Projects 1 and 4, and Project Milwaukee. MIDUS is a multi-stage probability sample of community-based English-speaking adults (aged 25 to 74) from the United States. MIDUS 2 was carried out between 2004-2009 and recruited 5,555 participants. In the current study, we only included participants with measures of coping, depressive symptoms, and sleep. Sleep was assessed using the Pittsburgh Sleep Quality Index (PSQI) [45], a sleep diary, and actigraphy. Of the 5,555 participants invited to Midus 2 (Project 1), 1255 were asked to respond to questions on sleep and depression (Project 4). Moreover, 445 and 441 participants (out of these 1,255) completed a sleep diary and wore an actigraph for seven days, respectively. After the exclusion of participants with missing data for the variables of interest and covariates (see description of variables below), the sample included in this study consisted of 723 participants (mean age= 54.22, $\mathrm{SD}=11.84$; men= 330, women= 393) for the PSQI, 437 for sleep diaries, and 428 for the actigraphy data. Participants excluded from this study due to missing data had a lower educational level and a higher body mass index. They reported more symptoms and chronic conditions, and there were more females and males than in the group of participants included in the study $(p<.04)$. No differences in age, alcohol intake and use of sleep medication, depression, or coping and sleep measures were observed between individuals with and without missing data $(p>.09)$. The institutional review board for Human Subject Protection at the UWMadison approved the study protocol for data collection. A detailed description of the sample and the MIDUS 2 can be found on the website of the project (http://midus.wisc.edu). 


\section{Measures}

\section{Coping}

Dispositional emotion-focused coping and problem-focused coping were measured using the 24-item version of the Coping Orientation to Problems Experienced Inventory (COPE) [46], as in previous research $[47,48]$. Participants were asked to answer a series of questions about how they generally respond when they are confronted with difficult or stressful events in their life. The response scale ranged from 1 (A lot) to 4 (Not at all). The questionnaire has six subscales: "Focus on and venting of emotion", "Denial", "Behavioral disengagement", "Positive Reinterpretation and Growth", "Active Coping", and "Planning". Emotion-focused coping was calculated by combining "Focus on and venting of emotion", "Denial", and "Behavioral disengagement". Problem-focused coping was calculated by combining the subscales "Positive Reinterpretation and Growth", "Active Coping", and "Planning". The literature $[49,50]$ supports combining the items belonging to the active coping, planning, and positive reinterpretation and growth subscales into a problem-focused coping factor, and combining the items in the venting emotions, denial, and behavioral disengagement subscales into an emotion-focused or dysfunctional coping factor. The scores on emotion-focused coping and problem-focused coping range from $12-48$ in each case. Cronbach's alpha for the total sample was .83 for emotion-focused coping and .90 for problem-focused coping.

\section{Depression}

We measured depressive symptoms during the past week with the 20-item Center for Epidemiological Studies Depression Inventory (CES-D) [51,52], using a response scale ranging from 0 (rarely or none of the time) to 3 (most or all of the time). The global score on the CES-D ranges from 0-60. The threshold traditionally used to detect depression on the CES-D is a score of 16 or more $[52,53]$. Cronbach's alpha for this scale in the total sample was .89 . 
Sleep

Pittsburgh Sleep Quality Index. General subjective sleep measures were obtained using the PSQI [30], a self-administered questionnaire about usual sleep habits during the past month that includes seven subscales: "Sleep quality", "Sleep latency", "Sleep duration", "Habitual sleep efficiency", "Sleep disturbances", "Use of sleep medication", and "Daytime dysfunction". The global score on the PSQI ranges from 0-21, and it is calculated by combining the seven subscales. A global PSQI score above 5 distinguishes good sleepers from poor sleepers [45]. The Cronbach's alpha for this scale in the total sample was .70.

Actigraphy. Detailed descriptions of actigraphy data collection and scoring methods are publicly available at the Interuniversity Consortium for Political and Social Research (ICPSR) website (URL: http://www.icpsr.umich.edu). Briefly, the Mini Mitter ActiwatchH-64 activity monitor is a device designed to detect the number of movements with a built-in motion sensor. The participants wore the actiwatch on their non-dominant wrist for seven consecutive days and nights. The data collection began at 7:00 a.m. on the Tuesday following a visit to the study center, and it concluded the following Tuesday morning. The data were imported and analyzed using the Actiware 5 software, a software that detects sleep based on 30 sec epochs and uses the manufacturer's algorithms following the formula validated by Oakley [54]. A mediumsensitivity threshold was used for data evaluation. Three indexes were calculated from the actiwatch data: (i) Total sleep time during the night was used as an index of objective sleep quantity, and (ii) wake after sleep onset (WASO) and (iii) Sleep efficiency were computed as indexes of objective sleep quality, where normative levels of sleep efficiency start at $80 \%-85 \%$ [55]. Sleep time was operationalized as the total number of epochs during a given interval scored as sleep, multiplied by the epoch length in minutes. WASO was operationalized as the total number of epochs between the start time and the end time awake in the given sleep 
interval, multiplied by the epoch length in minutes. Finally, Sleep Efficiency was calculated as the percentage of scored total sleep time for a given period in bed.

Sleep Diary. On the same days the participants were wearing the actigraph, they were asked to answer several questions related to their sleep. Two indexes were calculated from the sleep diary: (i) the Daily perceived sleep quantity and (ii) the Daily perceived sleep quality. The Daily perceived sleep quantity (in hours) was calculated using the participants' responses to three questions asked every day upon awakening: What time did you go to bed and begin trying to go to sleep? How long did it take you to get to sleep last night? What time did you wake up for the day and not go back to sleep? To assess participants' Daily perceived sleep quality, we calculated a global index of perceived sleep quality on the basis of participants' responses to the following questions every day upon awakening: How well-rested do you feel this morning (1 - well-rested to 5 - poorly-rested)? How deeply did you sleep last night (1 - very deeply to 5 very lightly)? How alert do you feel this morning (1 - very alert to 5 - not alert at all)? What was the overall quality of your sleep last night (1 - very good to 5 - very poor)?

\section{Control variables}

Based on previous studies [56-58], we considered a series of variables that could have an influence on our variables of interest. For the analyses with PSQI, the following covariates were considered: age, sex (men $=1$; women $=2)$, body mass index (BMI; weight in kilograms divided by the square of height in meters), total number of symptoms and chronic conditions ever reported/diagnosed (heart disease, high blood pressure, circulation problems, blood clots, heart murmur, TIA or stroke, anemia or another blood disease, cholesterol problems, diabetes, asthma, emphysema/COPD, tuberculosis, positive TB skin test, thyroid disease, cancer, and peptic ulcer disease), highest level of education completed (Responses were grouped into 12 categories ranging from $1=$ "no school/some grade school" to $12=$ "PhD, MD, JD, or another 
professional degree"), use of sleep medication ( 1 = not during the past month; 2 = less than once a week; 3 = once or twice a week, 4 = three or more times a week), and frequency of drinking during the previous month ( 1 = every day; $2=5$ or 6 days a week; $3=3$ or 4 days a week; 4 = 1 or 2 days a week; 5 = less than one day a week; 6 = never drink, volunteered). For the analyses with actigraphy and sleep diary data, the following covariates were considered: age, sex, BMI, total number of symptoms and chronic conditions, highest level of education completed, whether participants took a nap on the day of the assessments $(1=$ no, $2=$ yes, we computed the mean for the whole week), whether they took sleep medication or supplements on the day of the assessment ( $1=$ no, $2=$ yes, we computed the mean for the whole week), mean number of caffeine units ( 1 cup $=1$ unit) on the assessment days, and mean number of alcohol units ( 1 unit = 1 bottle beer or wine cooler, 1 glass of wine, or 1 shot of liquor) on the assessment days.

\section{Statistical analyses}

Correlation analyses were used to investigate the relationships among the sleep indexes, coping strategies (emotion-focused coping and problem-focused coping), depression, and the covariates included in the study.

Mediation models were performed using hierarchical regression analyses of direct and indirect effects, and bootstrapped bias-corrected 95\% confidence intervals of the indirect effects were computed using the PROCESS macro (version 2.16.3) in SPSS (Model 4) with 5000 bootstrapped samples. Confidence intervals that do not contain zero indicate a significant effect. As independent variable, dependent variable, and mediator, we included coping (i.e., emotion-focused or problem-focused), sleep (i.e., PSQI, actigraphy or sleep diary data), and depression, respectively. The analyses were first performed without covariates. Then, all the mediation models were repeated including covariates. In order to estimate the effect sizes of 
significant indirect effects, we used the Completely Standardized Indirect Effect ( $a b_{c S}$ ) index. We also used the Percent Mediation $\left(P_{M}\right)$ index for the indirect and direct effects, which indicates the proportion of the indirect/direct effect compared to the total effect.

Outliers were defined as values $\pm 3 S D$ and winsorized by replacing their values with values equal to the mean $\pm 3 S D$. Given that the sample was different for each independent variable, different numbers of outliers were also detected: In the analyses with the PSQI, 1 outlier was detected for PSQI, 1 for Emotion-focused coping, 2 for Problem-focused coping, and 12 for Depression. In the analyses with actigraphy, 8 outliers were detected for WASO, 7 for sleep efficiency, 1 for Emotion-focused coping, 3 for Problem-focused coping, and 7 for Depression. In the analyses with the Sleep diary, 3 outliers were detected for perceived sleep time, 3 for perceived sleep quality, 1 for emotion-focused coping, 2 for Problem-focused coping, and 7 for Depression. Statistical analyses were carried out using SPSS v.25 [59].

\section{Results}

\section{Characteristics of the study sample}

The characteristics of the study sample are shown in Table 1. There were 351 participants (48.5\%) who met the clinical PSQI cutpoint of beyond 5 that distinguishes good from bad sleepers. Also, 113 participants (15.63\%) scored at or above the cutoff score of 16, traditionally used to detect depression [52]. Finally, using $80 \%$ and $85 \%$ as clinical markers of normative levels of sleep efficiency [55], there are 174 participants (40.65\%) with sleep efficiency below $80 \%$ and 287 participants (67.06\%) with sleep efficiency below $85 \%$.

\section{Unadjusted Correlation Analyses}

Correlations between study variables are displayed in Table 2. Depression was positively related to emotion-focused coping and negatively related to problem-focused coping (both $p s<$ $.01)$, and there was a negative association between emotion-focused and problem-focused 
coping $(p<.01)$. The PSQI was positively related to emotion-focused coping and depression (both $p s<.01)$, and negatively related to problem-focused coping $(p<.05)$. None of the actigraphy indexes were significantly related to Problem-focused coping (all $p s>.05$ ), and only WASO was positively related to emotion-focused coping $(p<.01)$. All the actigraphy measures were significantly related to depression: WASO positively $(p<.01)$, and sleep time and sleep efficiency negatively ( $p s<.05$ and $<.01$, correspondingly). Regarding the sleep diary, perceived (lack of) sleep quality was positively associated with emotion-focused coping $(p<.05)$ and depression $(p<.01)$, and negatively related to problem-focused coping $(p<.01)$. In addition, perceived sleep time was positively related to emotion-focused coping $(p<.05)$. Finally, there was a positive relationship between actigraphy-measured sleep time and perceived sleep time $(p<.01)$, as well as a positive relationship between perceived sleep quality and actigraphymeasured WASO $(p<.05)$ and a negative relationship between perceived sleep quality and actigraphy-measured sleep efficiency $(p<.01)$.

\section{Mediation Analyses}

In the next step, we investigated the hypothesized mediation effects between coping strategies (emotion- and problem-focused) and sleep, through depression. All the mediation results are displayed in Tables 3 and 4. In the text, we only report the statistically significant results.

\section{Emotion-Focused Coping}

Pittsburgh Sleep Quality Index. The results show an indirect effect of emotion-focused coping on PSQI $\left(\mathrm{B}=.14\right.$; IC $\left.95 \%=[.103, .193] ; a b_{C S}=.144[.103,191], P_{M \text { ind }}=.735\right)$ that is maintained after controlling for all covariates. Specifically, emotion-focused coping is significantly related to depressive symptoms ( $B=.30,95 \% \mathrm{Cl}[.231, .363])$, and depressive symptoms are significantly related to the PSQI ( $B=.49,95 \% \mathrm{Cl}[.415, .557])$. The results indicate 
a significant full mediation between emotion-focused coping and the PSQI through depressive symptoms.

Actigraphy. The findings show significant indirect effects from emotion-focused coping through depressive symptoms to actigraphy measures of sleep quality (i.e., sleep efficiency, WASO). Specifically, the results show (a) a significant mediation between emotion-focused coping and sleep efficiency through depressive symptoms $\left(\mathrm{B}=-.08 ; \mathrm{IC} 95 \%=[-.127,-.042] ; a b_{\mathrm{CS}}=\right.$ $\left.-.081[-.132,-.044] ; P_{M \text { ind }}=.921\right)$, where emotion-focused coping is significantly related to depressive symptoms ( $B=.31,95 \% \mathrm{Cl}[.222, .402])$ and depressive symptoms are significantly related to sleep efficiency $(B=-.25,95 \% \mathrm{Cl}[-.345,-.156])$, as well as (b) a significant mediation between emotion-focused coping and WASO through depression $(B=.05 ;$ IC $95 \%=[.022, .098]$; $\left.a b_{c s}=.057[.023, .102] ; P_{M \text { ind }}=.497\right)$, where emotion-focused coping is significantly related to depressive symptoms $(B=.31,95 \% \mathrm{Cl}[.222, .402])$ and depressive symptoms are significantly related to WASO $(B=.17,95 \% \mathrm{Cl}[.078, .266])$. That is, a greater use of emotion-focused coping was associated with more depressive symptoms, leading to worse objective sleep quality (i.e., higher WASO and lower sleep efficiency). These results remain significant after controlling for covariates. We also observed an indirect effect of emotion-focused coping on total sleep time $\left(\mathrm{B}=-.04 ; \mathrm{IC} 95 \%=[-.083,-.014] ; a b_{\mathrm{CS}}=-.044[-.084,-.013] ; P_{M \text { ind }}=-.622\right)$. Specifically, emotionfocused coping is significantly related to depressive symptoms ( $B=.31,95 \% \mathrm{Cl}[.222, .402])$, and depressive symptoms are significantly related to total sleep time $(B=-.14,95 \% \mathrm{Cl}[-.240,-.041])$. These results indicate that a greater use of emotion-focused coping was associated with more depressive symptoms, leading to less objective sleep quantity. However, this mediation does not remain significant after controlling for covariates.

Sleep Diary. A significant indirect effect is observed for the relationship between emotion-focused coping and perceived sleep quality through depression $(B=.15 ; \mathrm{IC} 95 \%=[.100$, 
$\left..210] ; a b_{C S}=.149[.100, .205] ; P_{M \text { ind }}=.826\right)$. Specifically, emotion-focused coping is significantly related to depressive symptoms $(B=.33,95 \% \mathrm{Cl}[.237, .415])$, and depressive symptoms are significantly related to perceived sleep quality $(B=.46,95 \% \mathrm{Cl}[.370, .548])$. That is, a greater use of emotion-focused coping is associated with more depressive symptoms, leading to worse subjective sleep quality. Moreover, the results also show a direct effect of emotion-focused coping on perceived sleep time $\left(\mathrm{B}=.13\right.$; IC $\left.95 \%=[.044, .214] ; P_{M \text { dir }}=.893\right)$ indicating that there is a direct relationship between a greater use of emotion-focused coping and more perceived sleep time. These results were maintained when controlling for the covariates.

\section{Problem-Focused Coping}

Pittsburgh Sleep Quality Index. The mediation analysis indicates a significant indirect effect between problem-focused coping and PSQI through depression $(B=-.14 ;$ IC 95\% = [-.179, $\left..098] ; a b_{C S}=-.136[-.178,-.099] ; P_{M \text { ind }}=1.834\right)$. Specifically, problem-focused coping is significantly related to depressive symptoms ( $B=-.26,95 \% \mathrm{Cl}[-.327,-.194])$, and depressive symptoms are significantly related to PSQI $(B=.52,95 \% \mathrm{Cl}[.451, .591])$. This means that a greater use of problem-focused coping was associated with fewer depressive symptoms, leading to better general sleep quality (i.e., lower score on the PSQI). These results remained unchanged after controlling for covariates.

Actigraphy. The findings indicate significant indirect effects from problem-focused coping through depression to actigraphy measures of sleep quality (i.e., sleep efficiency, WASO). Specifically, there was a significant indirect effect (a) between problem-focused coping and sleep efficiency through depression $\left(\mathrm{B}=.06\right.$; IC 95\% $[.035, .103] ; \mathrm{ab}_{\mathrm{cs}}=.065[.037, .106] ; P_{M}$ ind $=9.834$ ), where problem-focused coping is significantly related to depressive symptoms ( $B=-$ $.24,95 \% \mathrm{Cl}[-.330,-.145])$ and depressive symptoms are significantly related to sleep efficiency ( $B=-.27,95 \% \mathrm{Cl}[-.358,-174])$, as well as (b) between problem-focused coping and WASO 
through depression (B=-.05; IC 95\% [-.084, -.021]; $\left.\mathrm{ab}_{\mathrm{CS}}=-.048[-.087,-.022] ; P_{M \text { ind }}=1.505\right)$, where problem-focused coping is significantly related to depressive symptoms $(B=-.24,95 \% \mathrm{Cl}$ $[-.330,-.145])$ and depressive symptoms are significantly related to WASO $(B=.19,95 \% \mathrm{Cl}[.101$, .284]). These results indicate that a greater use of problem-focused coping was associated with fewer depressive symptoms, leading to better objective sleep quality (i.e., lower WASO and higher sleep efficiency), and they remained significant after controlling for covariates. We also observed an indirect effect of problem-focused coping on the actigraphy-measured sleep time (B=.03; IC 95\% [.006, .060]; $\left.a b_{\mathrm{CS}}=.028[.006, .061] ; P_{M \text { ind }}=-.886\right)$. Specifically, problem-focused coping is significantly related to depressive symptoms ( $B=-.24,95 \% \mathrm{Cl}[-.330,-.145])$, and depressive symptoms are significantly related to total sleep time $(B=-.12,95 \% \mathrm{Cl}[-.216,-.021])$. This indicates that a greater use of problem-focused coping is associated with fewer depressive symptoms, leading to more objective sleep quantity. However, the effect is no longer significant after including the covariates.

Sleep Diary. The results for the sleep diary show a partial mediation of depression in the relationship between problem-focused coping and perceived sleep quality (total effect $B=-28$; IC 95\% [-.369, -.187]; $\left.a b_{C S}=-.10 .[-.142,-.057] ; P_{M \text { ind }}=.35\right)$. Specifically, problem-focused coping is significantly related to depressive symptoms ( $B=-.23,95 \% \mathrm{Cl}[-.319,-.136])$, and depressive symptoms are significantly related to perceived sleep quality $(B=.43,95 \% \mathrm{Cl}[.344$, .513]). These results indicate that a greater use of problem-focused coping was associated with fewer depressive symptoms, leading to better subjective sleep quality. These results are maintained after controlling for covariates.

We have carried out additional analyses including sex as moderating variable in all our mediation analyses performed with PROCESS (v2.16.2 Model 59). We found only one significant interaction effect of problem-focused coping and sex on AW sleep time. Specifically, in men, 
there was a significant direct effect of problem-focused coping on AW Sleep Time, whereas this direct effect was not significant in women. We also found a significant interaction effect of depressive symptoms and sex on sleep efficiency. However, this effect was no longer present when including covariates in the analyses. In addition, in order to rule out the possible moderating role of age in all the proposed mediations, we carried out moderated mediation analyses performed with PROCESS (v2.16.2 Model 59) with the variable age as moderator. The results show that there was no significant interaction effect of age on the direct/indirect/total effects.

\section{Discussion}

The purpose of this study was to investigate the relationship between coping strategies (i.e., emotion-focused and problem-focused coping) and sleep (i.e., quantity and quality, perceived, and actigraphy-measured), and study whether this relationship is direct or mediated by depressive symptoms. The results show significant indirect effects from emotion-focused coping through depressive symptoms to: (a) general sleep quality (i.e., PSQI score); (b) actigraphy-measured sleep quality (i.e., sleep efficiency, WASO); and (c) subjective sleep quality. In all these cases, more emotion-focused coping was associated with more depressive symptoms, which, in turn, were associated with worse sleep. Moreover, the results showed that there is a direct positive relationship between the use of emotion-focused coping strategies and perceived sleep time. Regarding problem-focused coping, the results showed significant indirect effects from problem-focused coping through depressive symptoms to: (a) general sleep quality (i.e., PSQI score); (b) actigraphy-measured sleep quality (i.e., sleep efficiency, WASO); and (c) subjective sleep quality. In all cases, more problem-focused coping was associated with having fewer depressive symptoms, which, in turn, was associated with better subjective and objective sleep parameters. 
This study provides strong evidence for the critical role of depression in the relationship between coping and sleep quality. In line with previous studies [8,22-25], unadjusted correlation analyses showed a significant relationship between emotion-focused coping and worse sleep (specifically, PSQI, WASO, and perceived lack of sleep in a sleep diary). However, the results of the mediation models explain that, when depression is considered, the connection between emotion-focused coping and sleep quality occurs because of the impact of coping on depressive symptoms [30-33], a variable that has a strong connection with sleep problems [38-40]. Therefore, it is possible that the relationship between emotion-focused coping and sleep observed in previous studies was due to the fact that these studies did not consider the role of depressive symptoms. Overall, this study supports the idea that the emotion-focused coping style is an important factor in understanding the interindividual differences in sleep quality $[8,23,41]$, but it indicates that depression plays a central role in this relationship.

Furthermore, as observed with emotion-focused coping, our mediation results show that problem-focused coping is related to better sleep quality (PSQI score, WASO, sleep efficiency, and subjective sleep quality in diaries) through its relationship with depression. That is, a greater use of problem-focused coping is related to less depression, which, in turn, is associated with fewer sleep problems. These results indicate that problem-focused coping is an effective and positive strategy that can counterbalance the severity of depressive symptoms [34] and their effects on sleep [38-40].

Although our study was well powered and we used different measures of sleep quantity, most of the relationships with sleep quantity were not statistically significant after controlling for covariates. In addition, although sex and age have been considered important factors in the development of depression and insomnia, our results indicate that sex and age do not 
moderate most of the relationships between coping, depression, and sleep. Specifically, in men, but not in women, only problem-focused coping was associated with more objective sleep time (assessed using AW). However, this result was not replicated in other sleep markers.

Considering the large sample size, these results indicate that our findings are consistent across sex and the lifespan.

It is noteworthy that there was a significant but moderate negative correlation between problem-focused coping and emotion-focused coping. This result is conceptually meaningful and consistent with previous research suggesting that people can deal with stress by employing a relatively wide range of coping strategies [46]. Moreover, the possibility of empirically separating the coping strategies "means that it should be possible to study their effects separately" [46]. It is also interesting that emotion-focused coping and problem-focused coping correlated with distinct indicators of sleep. They both correlated (positively and negatively) with the PSQI. However, emotion-focused coping was positively related to WASO and perceived sleep time, whereas a greater use of problem-focused coping was related to better perceived sleep quality. Although the positive correlation between emotion-focused coping and perceived sleep time may seem counterintuitive at first glance, this significant association could be due to the fact that the participants may have noticed a longer time between falling asleep and awakening, being unaware, however, of (micro) awakenings during the night (a symptom of lack of sleep quality) that add to the time comprised in between. This idea is supported by the results indicating that emotion-focused coping correlates positively with WASO. In turn, the negative correlation between problem-focused coping and sleep quality might be explained by the fact that problem-focused coping can entail increased positive emotions (e.g., due to successful accomplishment of tasks or dealing with a stressor [60]), which might provoke positive retrospective bias when evaluating past events, such as the quality of sleep the 
previous night. Further research is needed to further investigate the association between different coping strategies and the specific sleep markers.

The contribution of this study is that, to our knowledge, it is the first to address the existence of a possible mediation path from coping through depression to sleep in a large community sample, considering different dimensions of coping (i.e., emotional and problemfocused) and facets of sleep (i.e., sleep quantity and quality), and employing different measures of sleep (i.e., general perceived sleep, perceived sleep using a diary, and actigraphy). Therefore, this study provides evidence of the relationship between coping, depression, and sleep. Furthermore, this study makes a clear contribution to the Psycho-bio-behavioral model of vulnerability to insomnia [8]. Specifically, it indicates that depression may be an important mediator in the relationship between coping and sleep problems, and it suggests that depression should be included in the model. It also indicates that the Psycho-bio-behavioral model of vulnerability to insomnia [8] should be expanded to include problem-focused coping.

The direction of the relationship between coping, depression, and sleep warrants discussion. Although our hypothesis is based on the idea proposed by the Psycho-biobehavioral model of vulnerability to insomnia that coping may lead to sleep problems [8], due to the cross-sectional design, this study cannot provide insight into causality between coping, depression, and sleep. In this context, previous research demonstrated that sleep problems may contribute to the development of depression (for a review see Fang et al. [61]). Moreover, it has been shown that changes in mood may affect the way coping strategies are used, and that some coping strategies observed in healthy individuals may change when people are depressed [62-65]. Thus, some alternative explanations can be proposed for our findings, such as the possibility that sleep problems lead to depression, which in turn leads to changes in coping, or that depressive symptoms lead to both the use of certain coping strategies more 
than others and worse sleep. Future studies employing longitudinal designs are clearly needed to shed light on the direction of the relationships observed in this study.

Apart from the novel findings and methodological strengths, some limitations should be considered. One limitation of this study is the use of actigraphy instead of polysomnography, which is considered the "gold standard" in sleep measurement. However, actigraphy is considered a reliable tool for examining sleep patterns in insomnia patients and for diagnosing circadian rhythm disorders $[66,67]$. Additionally, actigraphy is more practical than PSG because it makes it possible to conveniently record continuously for days [66]. Another limitation is the inclusion of only English-speaking subjects in the study. Moreover, most of the participants were White (82.99\%), and $95.1 \%$ had at least a high school education. Therefore, the generalizability of the results may be limited, and more research is needed with different populations. Finally, it is important to note that the effectiveness of coping strategies can be context dependent. For instance, emotion-focused coping strategies may be adaptive when responding to uncontrollable stressors or major life events $[17,18]$. Future studies are needed to investigate whether the relationship between coping strategies and sleep depends on the kinds of stressors to which individuals are exposed.

To conclude, this is the first study to show, in a large sample, a mediation path from coping through depression to sleep, while considering: (a) key dimensions of coping (i.e., emotion- and problem-focused); (b) different facets of sleep (i.e., sleep duration, sleep quality); and (c) and different measures of sleep (i.e., general sleep, perceived sleep using a diary, and actigraphy). We observed that depression is a critical factor in understanding the relationship between coping strategies and sleep quality. 


\section{References}

1. Felder JN, Epel ES, Coccia M, Puterman E, Prather AA. Effects of daily maladaptive coping on nightly sleep in mothers. Psychol Health. 2018;33(1):144-157.

doi:10.1080/08870446.2017.1310863

2. Zhai L, Zhang H, Zhang D. Sleep duration and depression among adults: A meta-analysis of prospective studies. Depress Anxiety. 2015;32(9):664-670. doi:10.1002/da.22386

3. Lim ASP, Kowgier M, Yu L, Buchman AS, Bennett DA. Sleep fragmentation and the risk of incident Alzheimer's disease and cognitive decline in older persons. Sleep.

2013;36(7):1027-1032. doi:10.5665/sleep.2802

4. Bruno RM, Palagini L, Gemignani A, et al. Poor sleep quality and resistant hypertension. Sleep Med. 2013;14(11):1157-1163. doi:10.1016/j.sleep.2013.04.020

5. Hasler G, Buysse DJ, Klaghofer R, et al. The association between short sleep duration and obesity in young adults: A 13-year prospective study. Sleep. 2004;27(4):661-666.

doi:10.1093/sleep/27.4.661

6. Espie CA. Insomnia: Conceptual Issues in the development, persistence, and treatment of sleep disorder in adults. Annu Rev Psychol. 2002;53(1):215-243.

doi:10.1146/annurev.psych.53.100901.135243

7. Morin $\mathrm{CM}$, Rodrigue $\mathrm{S}$, Ivers $\mathrm{H}$. Role of stress, arousal, and coping skills in primary insomnia. Psychosom Med. 2003;65(2):259-267.

doi:10.1097/01.PSY.0000030391.09558.A3

8. Harvey C-J, Gehrman P, Espie CA. Who is predisposed to insomnia: A review of familial aggregation, stress-reactivity, personality and coping style. Sleep Med Rev. 2014;18(3):237-247. doi:10.1016/j.smrv.2013.11.004

9. Compas BE, Connor-Smith JK, Saltzman H, Thomsen AH, Wadsworth ME. Coping with stress during childhood and adolescence: Problems, progress, and potential in theory and research. Psychol Bull. 2001;127(1):87-127. doi:10.1037//0033-2909.127.1.87

10. Coppens CM, de Boer SF, Koolhaas JM. Coping styles and behavioural flexibility: Towards underlying mechanisms. Philos Trans R Soc Lond B Biol Sci. 2010;365(1560):4021-4028. doi:10.1098/rstb.2010.0217

11. Ben-Zur H. Transactional Model of Stress and Coping. In: Zeigler-Hill V, Shackelford TK, eds. Encyclopedia of Personality and Individual Differences. Cham: Springer International Publishing; 2019:1-4. doi:10.1007/978-3-319-28099-8_2128-1

12. Carver CS, Connor-Smith J. Personality and coping. Annu Rev Psychol. 2010;61(1):679704. doi:10.1146/annurev.psych.093008.100352 
13. Dewe PJ. Examining the nature of work stress: Individual evaluations of stressful experiences and coping. Hum Relat. 1989;42(11):993-1013.

doi:10.1177/001872678904201103

14. González-Morales MG, Rodríguez I, Peiró JM. A longitudinal study of coping and gender in a female-dominated occupation: Predicting teachers' burnout. J Occup Health Psychol. 2010;15(1):29-44. doi:10.1037/a0018232

15. Cooper CL, Dewe PJ, O'Driscoll MP. Organizational Stress: A Review and Critique of Theory, Research, and Applications. Thousand Oaks, CA: Sage Publication, Inc; 2001:xiv, 270.

16. Griffith J, Steptoe A, Cropley M. An investigation of coping strategies associated with job stress in teachers. Br J Educ Psychol. 1999;69(4):517-531. doi:10.1348/000709999157879

17. Park CL. Implicit religion and the meaning making model. Implicit Relig. 2011;14(4):405419. doi:10.1558/imre.v14i4.405

18. Strentz T, Auerbach SM. Adjustment to the stress of simulated captivity: Effects of emotion-focused versus problem-focused preparation on hostages differing in locus of control. J Pers Soc Psychol. 1988;55(4):652-660. doi:10.1037/0022-3514.55.4.652

19. Vitaliano PP, DeWolfe DJ, Maiuro RD, Russo J, et al. Appraised changeability of a stressor as a modifier of the relationship between coping and depression: A test of the hypothesis of fit. J Pers Soc Psychol. 1990;59(3):582-592. doi:10.1037//0022-3514.59.3.582

20. Aldwin CM. Stress, Coping, and Development: An Integrative Approach. New York, NY: Guilford press; 2007.

21. Rodríguez I, Kozusznik MW, Peiró JM, Tordera N. Individual, co-active and collective coping and organizational stress: A longitudinal study. Eur Manag J. 2019;37(1):86-98. doi:10.1016/j.emj.2018.06.002

22. Hicks RA, Marical CM, Conti PA. Coping with a major stressor: Differences between habitual short- and longer-sleepers. Percept Mot Skills. 1991;72(2):631-636. doi:10.2466/PMS.72.2.631-636

23. Sadeh A, Keinan G, Daon K. Effects of stress on sleep: The moderating role of coping style. Health Psychol. 2004;23(5):542-545. doi:10.1037/0278-6133.23.5.542

24. Hoyt MA, Thomas KS, Epstein DR, Dirksen SR. Coping style and sleep quality in men with cancer. Ann Behav Med. 2009;37(1):88-93. doi:10.1007/s12160-009-9079-6

25. Fernández-Mendoza J, Vela-Bueno A, Vgontzas AN, et al. Cognitive-emotional hyperarousal as a premorbid characteristic of individuals vulnerable to insomnia. Psychosom Med. 2010;72(4):397-403. doi:10.1097/PSY.0b013e3181d75319 
26. Compas BE. Psychobiological processes of stress and coping: implications for resilience in childhood and adolescence. Ann N Y Acad Sci. 2006; 1094: 226-234.

27. Holahan CJ, Moos RH, Schaefer J. Coping, resilience, and growth: Conceptualizing adaptive functioning. In: Zeidner M, Endler N, eds. Handbook of Coping: Research, Theory, and Application. New York: Wiley; 1996:24-43.

28. Moos RH, Holahan CJ. Dispositional and contextual perspectives on coping: Toward an integrative framework. J Clin Psychol. 2003; 59: 1387-1403.

29. Beck AT, Rush AJ, Shaw BF, Emery G. Cognitive Therapy of Depression. New York, NY: Guilford press; 1979.

30. Burker EJ, Evon D, Loiselle MM, Finkel J, Mill M. Planning helps, behavioral disengagement does not: Coping and depression in the spouses of heart transplant candidates. Clin Transplant. 2005;19(5):653-658. doi:10.1111/j.1399-0012.2005.00390.x

31. Klostermann K, Chen R, Kelley ML, Schroeder VM, Braitman AL, Mignone T. Coping Behavior and depressive symptoms in adult children of alcoholics. Subst Use Misuse. 2011;46(9):1162-1168. doi:10.3109/10826080903452546

32. Kramer BJ. Expanding the conceptualization of caregiver coping: The importance of relationship-focused coping strategies. Fam Relat. 1993;42(4):383. doi:10.2307/585338

33. Zuckerman M, Gagne M. The COPE revised: Proposing a 5-factor model of coping strategies. J Res Pers. 2003;37(3):169-204. doi:10.1016/S0092-6566(02)00563-9

34. Bardwell WA, Ancoli-Israel S, Dimsdale JE. Types of coping strategies are associated with increased depressive symptoms in patients with obstructive sleep apnea. Sleep.

2001;24(8):905-909. doi:10.1093/sleep/24.8.905

35. Berghuis JP, Stanton AL. Adjustment to a dyadic stressor: A longitudinal study of coping and depressive symptoms in infertile couples over an insemination attempt. $J$ Consult Clin Psychol. 2002;70:433-438. doi:10.1037/0022-006X.70.2.433

36. Evans LD, Kouros C, Frankel SA, McCauley E, Diamond GS, Schloredt KA, Garber J. Longitudinal relations between stress and depressive symptoms in youth: Coping as a mediator. J Abnorm Child Psychol. 2015; 43: 355-368.

37. Kennedy P, Marsh N, Lowe R, Grey N, Short E, Rogers B. A longitudinal analysis of psychological impact and coping strategies following spinal cord injury. Br J Health Psychol. 2000; 5: 157-172.

38. Nicassio PM, Ormseth SR, Kay M, et al. The contribution of pain and depression to selfreported sleep disturbance in patients with rheumatoid arthritis: Pain. 2012;153(1):107112. doi:10.1016/j.pain.2011.09.024 
39 Gebara MA, Siripong N, DiNapoli EA, et al. Effect of insomnia treatments on depression: A systematic review and meta-analysis. Depress Anxiety. 2018;35(8):717-731. doi:10.1002/da.22776

40. Ohayon MM. Epidemiology of insomnia: what we know and what we still need to learn. Sleep Med Rev. 2002;6(2):97-111. doi:10.1053/smrv.2002.0186

41. Thomas KS, Bower J, Hoyt MA, Sepah S. Disrupted sleep in breast and prostate cancer patients undergoing radiation therapy: The role of coping processes. Psychooncology. 2010;19(7):767-776. doi:10.1002/pon.1639

42. Priester MJ, Clum, GA. Perceived problem-solving ability as a predictor of depression, hopelessness, and suicide ideation in a college population. J Couns Psychol. 1993;40:7985. doi:10.1037/0022-0167.40.1.79

43. Matheson K, Anisman H. Systems of Coping Associated with Dysphoria, Anxiety and Depressive Illness: A Multivariate Profile Perspective. Stress. 2003;6: 223-234. doi: $10.1080 / 10253890310001594487$

44. Ciarrochi J, Scott G. The link between emotional competence and well-being: a longitudinal study. Brit J Guid Couns. 2006; 34: 231-243. doi: $\underline{10.1080 / 03069880600583287}$

45. Buysse DJ, Reynolds CF, Monk TH, Berman SR, Kupfer DJ. The Pittsburgh sleep quality index: A new instrument for psychiatric practice and research. Psychiatry Res. 1989;28(2):193-213. doi:10.1016/0165-1781(89)90047-4

46. Carver CS, Scheier MF, Weintraub JK. Assessing coping strategies: A theoretically based approach. J Pers Soc Psychol. 1989;56(2):267-283.

47. Vassillière $\mathrm{CT}$, Holahan $\mathrm{CJ}$, Holahan CK. Race, perceived discrimination, and emotionfocused coping: Race and Emotion-Focused Coping. J Community Psychol. 2016;44(4):524-530. doi:10.1002/jcop.21776

48. Moran KM, Turiano NA, Gentzler AL. Parental warmth during childhood predicts coping and well-being in adulthood. J Fam Psychol. 2018;32(5):610-621. doi:10.1037/fam0000401

49. Kling KC, Seltzer MM, Ryff CD. Distinctive late-life challenges: Implications for coping and well-being. Psychol Aging. 1997;12(2):288-295. doi:10.1037/0882-7974.12.2.288

50. Snell DL, Siegert RJ, Hay-Smith EJC, Surgenor L. Factor structure of the Brief COPE in people with mild traumatic brain injury. J Head Trauma Rehabil. 2011;26(6):468-477. doi:10.1097/HTR.0b013e3181fc5e1e

51. Devins GM, Orme CM. Center for epidemiological studies depression scale. In: Keyser DJ, Sweetland RC, eds. Test Critiques. Kansas City, MO: Test Corporation America; 1985:144160. 
52. Radloff LS. The CES-D scale: A self-report depression scale for research in the general population. Appl Psych Meas. 1977;1(3):385-401.

53. APA American Psychiatric Association. Diagnostic and Statistical Manual of Mental Disorders, 4th ed. Washington, DC: American Psychiatric Association; 1994.

54. Oakley NR. Validation with Polysomnography of the Sleepwatch Sleep/Wake Scoring Algorithm Used by the Actiwatch Activity Monitoring System. Bend, OR: Mini-Mitter; 1997.

55. Morin CM, Hauri PJ, Espie CA, Spielman AJ, Buysse DJ, Bootzin RR. Nonpharmacologic treatment of chronic insomnia. Sleep. 1999; 22: 1134-1156. doi:10.1093/sleep/22.8.1134

56. Lauderdale DS, Knutson KL, Yan LL, et al. Objectively measured sleep characteristics among early-middle-aged adults. Am J Epidemiol. 2006;164(1):5-16.

doi:10.1093/aje/kwj199

57. Bei B, Wiley JF, Trinder J, Manber R. Beyond the mean: A systematic review on the correlates of daily intraindividual variability of sleep/wake patterns. Sleep Med Rev. 2016;28:108-124. doi:10.1016/j.smrv.2015.06.003

58. Morgan E, Schumm LP, McClintock M, Waite L, Lauderdale DS. Sleep characteristics and daytime cortisol levels in older adults. Sleep. 2017;40(5):zsx043.

doi:10.1093/sleep/zsx043

59. IBM Corp. IBM SPSS Statistics for Macintosh, Version 25. Armonk, NY: IBM Corp.; 2017.

60. Folkman S, Moskowitz JT. Stress, Positive Emotion, and Coping. Curr Dir Psychol Sci. 2000;9(4):115-118. doi:10.1111/1467-8721.00073

61. Fang $\mathrm{H}, \mathrm{Tu} \mathrm{S}$, Sheng J, Shao A. Depression in sleep disturbance: A review on a bidirectional relationship, mechanisms and treatment. J Cell Mol Med. 2019; 23(4):23242332.

62. Beck AT. Cognitive therapy of depression: New perspectives. In: Clayton PJ, Barrett JE, eds. Treatment of Depression: Old Controversies and New Approaches. New York, NY: Raven; 1983: 265-290.

63. Blatt SJ. Experiences of Depression: Theoretical, Clinical, and Research Perspectives. Washington, DC: American Psychological Association; 2004.

64. Dunkley DM, Ma D, Lee IA, Preacher KJ, Zuroff DC. Advancing complex explanatory conceptualizations of daily negative and positive affect: Trigger and maintenance coping action patterns. J Couns Psychol. 2014; 61: 93-109. doi: 10.1037/a0034673

65. Dunkley DM, Lewkowski M, Lee IA, Preacher KJ, Zuroff DC, Berg JL, Foley E, Myhr G, Westreich R. Daily stress, coping, and negative and positive affect in depression: Complex trigger and maintenance patterns. Behav Ther. 2017; 48: 349-365. 
66. Ancoli-Israel S, Cole R, Alessi C, Chambers M, Moorcroft W, Pollak CP. The role of actigraphy in the study of sleep and circadian rhythms. Sleep. 2003;26(3):342-392. doi:10.1093/sleep/26.3.342

67. Sadeh A, Acebo C. The role of actigraphy in sleep medicine. Sleep Med Rev. 2002;6(2):113-124. doi:10.1053/smrv.2001.0182 
Table 1. Descriptives

\begin{tabular}{|c|c|c|}
\hline Variable & $\mathrm{M} /$ count & (SD / \%) \\
\hline Age & 54.22 & $(11.84)$ \\
\hline \multicolumn{3}{|l|}{ Sex } \\
\hline Male & 330 & $(45.60 \%)$ \\
\hline Female & 393 & $(54.40 \%)$ \\
\hline \multicolumn{3}{|l|}{ Education } \\
\hline No school/some grade school (1-6) & 1 & $(0.10 \%)$ \\
\hline Eighth grade/junior high school (7-8) & 5 & $(0.70 \%)$ \\
\hline Some high school (9-12 no diploma/no GED) & 24 & $(3.30 \%)$ \\
\hline GED & 6 & $(0.80 \%)$ \\
\hline Graduated from high school & 138 & $(19.10 \%)$ \\
\hline 1 to 2 years of college, no degree yet & 118 & $(16.30 \%)$ \\
\hline 3 or more years of college, no degree yet & 32 & $(4.40 \%)$ \\
\hline Grad. From 2-year college, vocational school, or assoc. Degr & 46 & $(6.40 \%)$ \\
\hline Graduated from a 4- or 5-year college, or bachelor's degree & 171 & $(23.70 \%)$ \\
\hline Some graduate school & 36 & $(5.00 \%)$ \\
\hline Master's degree & 111 & $(15.40 \%)$ \\
\hline PH.D., ED.D., MD, DDS, LLB, LLD, JD, or other profess'nl deg & 35 & $(4.80 \%)$ \\
\hline \multicolumn{3}{|l|}{ 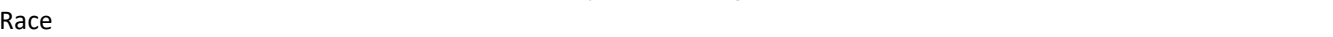 } \\
\hline White & 600 & (82.99\%) \\
\hline Black and/or African American & 98 & $(13.55 \%)$ \\
\hline Native American or Alaska native Aleutian Islander/Eskimo & 4 & $(0.55 \%)$ \\
\hline Asian & 1 & $(0.14 \%)$ \\
\hline Other & 18 & $(2.49 \%)$ \\
\hline Don't know & 2 & $(0.28 \%)$ \\
\hline Body Mass Index & 29.31 & (6.39) \\
\hline Total number of Symptoms and Chronic Conditions ${ }^{1}$ & 3.88 & $(2.83)$ \\
\hline \multicolumn{3}{|l|}{ Sleep medication } \\
\hline Not during the past month & 532 & (73.58\%) \\
\hline Less than once a week & 58 & $(8.02 \%)$ \\
\hline Once or twice a week & 33 & $(4.56 \%)$ \\
\hline Three or more times a week & 100 & $(13.83 \%)$ \\
\hline \multicolumn{3}{|l|}{ How often one drink past month } \\
\hline Every day & 74 & $(10.20 \%)$ \\
\hline 5 or 6 days a week & 51 & $(7.10 \%)$ \\
\hline 3 or 4 days a week & 95 & $(13.10 \%)$ \\
\hline 1 or 2 days a week & 165 & $(22.80 \%)$ \\
\hline Less than one day a week & 334 & (46.20\%) \\
\hline Never drink (volunteered) & 4 & $(0.60 \%)$ \\
\hline Average nap on assessments days ( $1=$ no, $2=$ yes $)$ & 1.74 & $(0.28)$ \\
\hline Sleep meds yesterday & 1.92 & $(0.24)$ \\
\hline Average caffeine units on the assessment days & 2.17 & (1.63) \\
\hline Average alcohol units on the assessment days & 0.83 & $(1.21)$ \\
\hline Depression (CES-D) & 8.62 & (8.14) \\
\hline Problem-focused coping (COPE) & 38.39 & $(5.80)$ \\
\hline Emotion-focused coping (COPE) & 22.11 & $(5.33)$ \\
\hline Pittsburgh Sleep Quality Index (PSQI) & 6.21 & (3.57) \\
\hline Perceived sleep quality (sleep diary) & 2.34 & (0.69) \\
\hline Perceived sleep time (sleep diary) & 7.54 & (1.09) \\
\hline Sleep efficiency (Actigraphy) & 80.49 & (9.56) \\
\hline WASO (Actigraphy) & 48.62 & (22.52) \\
\hline Sleep time (Actigraphy) & 378.00 & (65.37) \\
\hline
\end{tabular}

Note. $\mathrm{CES}-\mathrm{D}=$ Center for Epidemiological Studies Depression Inventory; COPE= Coping Orientation to Problems Experienced Inventory; PSQI=Pittsburg Sleep Quality Inventory; WASO=Wake after sleep onset. ${ }^{1}$ Total number of symptoms and chronic conditions: heart disease, high blood pressure, circulation problems, blood clots, heart murmur, TIA or stroke, anemia or other blood disease, cholesterol problems, diabetes, asthma, emphysema/COPD, tuberculosis, positive TB skin test, thyroid disease, cancer, and peptic ulcer disease. 
Table 2. Correlations among the study variables.

\begin{tabular}{|c|c|c|c|c|c|c|c|c|c|c|c|c|c|c|c|c|c|c|c|}
\hline Variable & (1) & $(2)$ & (3) & (4) & (5) & (6) & (7) & (8) & (9) & (10) & (11) & (12) & (13) & (14) & (15) & $(16)$ & (17) & (18) & (19) \\
\hline \multicolumn{20}{|l|}{ 1.Age } \\
\hline 2.Sex & -.09 & & & & & & & & & & & & & & & & & & \\
\hline 3. Education & .01 & -.06 & & & & & & & & & & & & & & & & & \\
\hline 4. Body Mass Index & -.06 & -.03 & -.12 & & & & & & & & & & & & & & & & \\
\hline $\begin{array}{l}\text { 5. Symptoms and chronic } \\
\text { conditions }\end{array}$ & .38 & .10 & -.01 & .13 & & & & & & & & & & & & & & & \\
\hline 6. Sleeping medication & .00 & .07 & .01 & .03 & .21 & & & & & & & & & & & & & & \\
\hline 7. Alcohol & -.11 & .24 & -.02 & .11 & -.07 & -.06 & & & & & & & & & & & & & \\
\hline 8. Naps today & -.14 & .12 & .06 & -.13 & -.11 & .00 & -.11 & & & & & & & & & & & & \\
\hline 9. Sleep meds yesterday & -.08 & -.09 & -.05 & .05 & -.26 & -.52 & .01 & .02 & & & & & & & & & & & \\
\hline 10. Caffeine today & -.08 & -.17 & .02 & -.01 & -.10 & -.11 & -.06 & .05 & .07 & & & & & & & & & & \\
\hline 11. Alcohol today & -.11 & -.23 & -.11 & -.08 & -.15 & -.05 & -.56 & .02 & .05 & .13 & & & & & & & & & \\
\hline $\begin{array}{l}\text { 12. Emotion-focused } \\
\text { coping }\end{array}$ & .01 & .16 & -.17 & -.01 & .16 & .14 & .03 & -.03 & -.17 & -.04 & .07 & & & & & & & & \\
\hline $\begin{array}{l}\text { 13. Problem-focused } \\
\text { coping }\end{array}$ & .06 & .11 & .05 & .02 & -.02 & .03 & .01 & .13 & .00 & -.01 & -.17 & -.25 & & & & & & & \\
\hline 14. Depression & -.16 & .03 & -.11 & .12 & .17 & .18 & -.01 & -.17 & -.06 & -.03 & -.03 & .30 & -.27 & & & & & & \\
\hline 15. PSQI & -.13 & .12 & -.08 & .09 & .24 & .55 & .01 & -.20 & -.33 & -.03 & .03 & .20 & -.08 & .48 & & & & & \\
\hline 16. Sleep time & .05 & .14 & -.01 & -.14 & -.13 & -.10 & .03 & .28 & .02 & -.01 & -.07 & .05 & -.06 & -.15 & -.29 & & & & \\
\hline 17. Sleep efficiency & .02 & .10 & .13 & -.23 & -.24 & -.26 & .11 & .26 & .04 & .15 & -.19 & -.13 & .04 & -.25 & -.39 & .64 & & & \\
\hline 18. WASO & .03 & -.04 & -.09 & .19 & .24 & .27 & -.09 & -.18 & -.05 & -.12 & .17 & .17 & -.07 & .18 & .32 & -.15 & -.69 & & \\
\hline $\begin{array}{l}\text { 19. Perceived sleep } \\
\text { quality }\end{array}$ & -.20 & -.03 & .02 & .06 & .08 & .14 & -.02 & -.13 & -.13 & -.08 & .07 & .13 & -.33 & .42 & .48 & -.09 & -.18 & .14 & \\
\hline 20. Perceived sleep time & .02 & .06 & -.15 & .03 & .03 & .13 & -.06 & .15 & -.05 & -.11 & .10 & .14 & -.09 & -.02 & -.10 & .68 & -.02 & .34 & -.04 \\
\hline
\end{tabular}

Note. Results in bold indicate a significant relationship ( $p<.05 ; 2$-tailed). 
Table 3. Mediation effects between emotion-focused coping and sleep through depression.

\begin{tabular}{|c|c|c|c|c|c|c|c|}
\hline & & $\mathrm{X}-\mathrm{M}$ & $M-Y$ & $X-Y$ & Indirect effect & Direct effect & Total effect \\
\hline \multirow[t]{2}{*}{ EC-Dep - PSQI } & Unadjusted & $.30^{*}$ & $.49^{*}$ & $.20^{*}$ & Est. $=.14, \mathrm{Cl}[.103 ; .193]$ & Est. $=.05, \mathrm{Cl}[-.015 ; .119]$ & Est. $=.20, \mathrm{Cl}[.124 ; .268]$ \\
\hline & Adjusted & $.26^{*}$ & $.38 *$ & $.08 *$ & Est. $=.10, \mathrm{Cl}[.066 ; .130]$ & Est. $=-.01, \mathrm{Cl}[-.073 ; .043]$ & Est. $=.08 ; \mathrm{Cl}[.021 ; .142]$ \\
\hline \multirow[t]{2}{*}{ EC-Dep-SE } & Unadjusted & $.31 *$ & $-.25 *$ & -.09 & Est. $=-.08, \mathrm{Cl}[-.127 ;-.042]$ & Est. $=-.01, \mathrm{Cl}[-.100 ; .087]$ & Est. $=-.08, \mathrm{Cl}[-.176 ; .007]$ \\
\hline & Adjusted & $.26^{*}$ & $-.17 *$ & -.07 & Est. $=-.04, \mathrm{Cl}[-.082 ;-.019]$ & Est. $=-.02, \mathrm{Cl}[-.115 ; .067]$ & Est. $=-.07, \mathrm{Cl}[-.158 ; .021]$ \\
\hline \multirow[t]{2}{*}{ EC-Dep - WASO } & Unadjusted & $.31 *$ & $.17^{*}$ & $.11^{*}$ & Est. $=.05, \mathrm{Cl}[.022 ; .098]$ & Est. $=.05, \mathrm{Cl}[-.039 ; .147]$ & Est.=.11, Cl $[.018 ; .197]$ \\
\hline & Adjusted & $.26^{*}$ & $.11^{*}$ & .07 & Est. $=.03, \mathrm{Cl}[.004 ; .065]$ & Est. $=.04, \mathrm{Cl}[-.054 ; .133]$ & Est. $=.07, \mathrm{Cl}[-.023 ; .158]$ \\
\hline \multirow[t]{2}{*}{ EC - Dep - ST } & Unadjusted & $.31^{*}$ & $-.14 *$ & .07 & Est. $=-.04, \mathrm{Cl}[-.083 ;-.014]$ & Est. $=.11, \mathrm{Cl}[.015 ; .213]$ & Est. $=.07, \mathrm{Cl}[-.024 ; .165]$ \\
\hline & Adjusted & $.26^{*}$ & -.08 & .05 & Est. $=-.02, \mathrm{Cl}[-.052 ; .004]$ & Est. $=.07, \mathrm{Cl}[-.030 ; .166]$ & Est. $=.05, \mathrm{Cl}[-.047 ; .142]$ \\
\hline \multirow[t]{2}{*}{ EC-Dep - PSQ } & Unadjusted & $.33^{*}$ & $.46^{*}$ & $.18^{*}$ & Est. $=.15, \mathrm{Cl}[.100 ; .210]$ & Est.=.03, Cl [-.057; .121] & Est.= .18, Cl [.088; .275] \\
\hline & Adjusted & $.27^{*}$ & $.42^{*}$ & $.14 *$ & Est. $=.11, \mathrm{Cl}[.072 ; .169]$ & Est. $=.02, \mathrm{Cl}[-.069 ; .115]$ & Est. $=.14, \mathrm{Cl}[.041 ; .233]$ \\
\hline \multirow{2}{*}{ EC-Dep-PST } & Unadjusted & $.33^{*}$ & .02 & $.14 *$ & Est. $=.01, \mathrm{Cl}[-.025 ; .046]$ & Est.=.13, Cl $[.044 ; .214]$ & Est.=.14, Cl [.056; .217] \\
\hline & Adjusted & $.27 *$ & .02 & $.10 *$ & Est. $=.01, \mathrm{Cl}[-.023 ; .039]$ & Est. $=.09, \mathrm{Cl}[.007 ; .180]$ & Est. $=.10, \mathrm{Cl}[.017 ; .183]$ \\
\hline
\end{tabular}

Note. EC=Emotion-focused coping; Dep=Depression; PSQI=Pittsburg Sleep Quality Inventory;

$\mathrm{SE}=$ Sleep efficiency; WASO=Wake after sleep onset; $\mathrm{ST}=$ Sleep time; $\mathrm{PSQ}=$ Perceived sleep quality; PST=Perceived sleep time $X=I n d e p e n d e n t$ Variable; $M=$ Mediator; $Y=$ Dependent Variable. *significant as per bootstrapped bias-corrected $95 \% \mathrm{Cl}$. 
Table 4. Mediation effects between problem-focused coping and sleep through depression

\begin{tabular}{|c|c|c|c|c|c|c|c|}
\hline & & $\mathrm{X}-\mathrm{M}$ & $M-Y$ & $X-Y$ & Indirect effect & Direct effect & Total effect \\
\hline \multirow[t]{2}{*}{ PC - Dep - PSQI } & Unadjusted & $-.26 *$ & $.52^{*}$ & $-.07^{*}$ & Est.=-.14, IC [-.179; -.098] & Est. $=.06, \mathrm{Cl}[-.005 ; .128]$ & Est. $=-.07, \mathrm{Cl}[-.147 ;-.001]$ \\
\hline & Adjusted & $-.25 *$ & $.38 *$ & $-.08 *$ & Est. $=-.09, \mathrm{Cl}[-.123 ;-.066]$ & Est. $=.01, \mathrm{Cl}[-.041 ; .070]$ & Est. $=-.08, \mathrm{Cl}[-.136 ;-.019]$ \\
\hline \multirow[t]{2}{*}{ PC-Dep-SE } & Unadjusted & $-.24 *$ & $-.27^{*}$ & .01 & Est.=.06, Cl [.035; .103] & Est.=-.06, Cl [-.149; .035] & Est.= .01, Cl [-.086; .099] \\
\hline & Adjusted & $-.19 *$ & $-.20 *$ & -.07 & Est. $=.04, \mathrm{Cl}[.017 ; .073]$ & Est. $=-.11, \mathrm{Cl}[-.195 ;-.019]$ & Est. $=-.07, \mathrm{Cl}[-.155 ; .020]$ \\
\hline \multirow{2}{*}{ PC - Dep - WASO } & Unadjusted & $-.24 *$ & $.19^{*}$ & -.03 & Est. $=-.05, \mathrm{Cl}[-.084 ;-.021]$ & Est. $=.02, \mathrm{Cl}[-.076 ; .107]$ & Est. $=-.03, \mathrm{Cl}[-.121 ; .060]$ \\
\hline & Adjusted & $-.19 *$ & $.13^{*}$ & .02 & Est. $=-.03, \mathrm{Cl}[-.056 ;-.007]$ & Est. $=.04, \mathrm{Cl}[-.050 ; .130]$ & Est. $=.02, \mathrm{Cl}[-.074 ; .104]$ \\
\hline \multirow[t]{2}{*}{ PC-Dep-ST } & Unadjusted & $-.24 *$ & $-.12^{*}$ & -.03 & Est. $=.03, \mathrm{Cl}[.006 ; .060]$ & Est. $=-.06, \mathrm{Cl}[-.157 ; .038]$ & Est. $=-.03, \mathrm{Cl}[-.127 ; .063]$ \\
\hline & Adjusted & $-.19 *$ & -.08 & -.08 & Est. $=.02, \mathrm{Cl}[-.001 ; .042]$ & Est. $=-.10, \mathrm{Cl}[-.191 ;-.002]$ & Est. $=-.08, \mathrm{Cl}[-.173 ; .012]$ \\
\hline \multirow[t]{2}{*}{ PC-Dep-PSQ } & Unadjusted & $-.23 *$ & $.43^{*}$ & $-.28 *$ & Est. $=-.10, \mathrm{Cl}[-.144 ;-.056]$ & Est. $=-.18, \mathrm{Cl}[-.265 ;-.096]$ & Est. $=-.28, \mathrm{Cl}[-.369 ;-.187]$ \\
\hline & Adjusted & $-.19 *$ & $.39 *$ & $-.25^{*}$ & Est. $=-.07, \mathrm{Cl}[-.118 ;-.037]$ & Est. $=-.18, \mathrm{Cl}[-.266 ;-.093]$ & Est. $=-.25, \mathrm{Cl}[-.345 ;-.162]$ \\
\hline \multirow[t]{2}{*}{ PC-Dep-PST } & Unadjusted & $-.23 *$ & .07 & -.01 & Est. $=-.02, \mathrm{Cl}[-.047 ; .009]$ & Est. $=.00, \mathrm{Cl}[-.083 ; .084]$ & Est. $=-.01, \mathrm{Cl}[-.096 ; .067]$ \\
\hline & Adjusted & $-.19 *$ & .05 & -.01 & Est. $=-.01, \mathrm{Cl}[-.035 ; .011]$ & Est. $=.00, \mathrm{Cl}[-.081 ; .086]$ & Est. $=-.01, \mathrm{Cl}[-.088 ; .075]$ \\
\hline
\end{tabular}

Note. PC=Problem-focused coping; Dep=Depression; PSQI=Pittsburg Sleep Quality Inventory; SE=Sleep efficiency; WASO=Wake after sleep onset; $S T=S l e e p$ time; $P S Q=P e r c e i v e d$ sleep quality; PST=Perceived sleep time; $\mathrm{X}=$ Independent Variable; $\mathrm{M}=$ Mediator; $\mathrm{Y}=$ Dependent Variable. * significant as per bootstrapped bias-corrected $95 \% \mathrm{Cl}$. 